\title{
A case of chylothorax due to primary renal lymphoma
}

\author{
Onur Turan ${ }^{1}$, Hayri Ozsan ${ }^{2}$, Oguz Dicle ${ }^{3}$, Atila Akkoclu ${ }^{1}$ \\ 1. Chest Diseases Department, Faculty of Medicine, Dokuz Eylul University, İzmir, Turkey. 2. Hematology Department, \\ Faculty of Medicine, Dokuz Eylul University, İzmir, Turkey. 3. Radiodiagnostics Department, Faculty of Medicine, Dokuz \\ Eylul University, İzmir, Turkey.
}

Correspondence: Onur Turan. Address: Chest Diseases Department, Faculty of Medicine, Dokuz Eylul University, İzmir , Turkey. Email: onurtura@yahoo.com

Received: February 7, 2014

DOI : $10.5430 /$ crim.v1n2p183

Online Published: June 17, 2014

URL: http://dx.doi.org/10.5430/crim.v1n2p183

\section{Abstract}

Chylothorax is a type of pleural effusion which develops by collection of lymphatic fluid in the pleural cavity after disruption or blockage of the thoracic duct.

The posterior-anterior lung graphy revealed a left pleural effusion during routine controls of an asymptomatic 79 year-old male patient. Pleural fluid with a chylous appearance drained by thoracentesis had a triglyceride level of $409 \mathrm{mg} / \mathrm{dL}$. As chylothorax was thought as diagnosis, thoracoabdominal computed tomography revealed a left renal mass and left pleural effusion. There was a mass lesion taking origin from the superior pole of the left kidney with multiple periaortic and perirenal lymphadenopathies in magnetic resonance imaging of the abdomen, which was interpreted as lymphoma. The tru-cut biopsy from renal lesion revealed as diffuse large B-cell non-Hodgkin lymphoma (NHL).

Non-traumatic chylothorax is most commonly due to malignancy. Lymphoma is the most common cause of chylothorax with approximately 75\%. Primary renal lymphoma, a type of NHL, has not ever found as a cause of chylothorax in our literature investigation. In this paper, we report this rarely seen case.

\section{Keywords}

Pleural effusion, Chylothorax, Primary renal lymphoma

\section{I ntroduction}

Chylothorax is the collection of lymphatic fluid in the pleural space after disruption or blockage of the thoracic duct or its major branches. Chyle is collected and transported by the thoracic duct into the circulation, so its damage or obstruction leads to leakage of chyle into the pleural space ${ }^{[1]}$. Chyle is a type of lymphatic fluid characterized by a white, milky appearance due to a high lipid content, consists of triglycerides.

As chylothorax has various causes, malignancy is known as the most common cause of chylothorax, with a frequency of $50 \%{ }^{[2]}$. Lymphoma, the most common reason of chylothorax in malignancies, occurs in approximately $75 \%$ of this group $^{[3]}$. 
Primary renal lymphoma, defined as a type of non Hodgkin's lymphoma, is known to originate in the renal parenchyma without the involvement of the kidney by primarily extrarenal lymphatic disease ${ }^{[4]}$. It is a rarely seen disease, which was found as $0.7 \%$ of all extra-nodal lymphomas and $0.1 \%$ of all malignant lymphomas ${ }^{[5]}$. As far as our knowledge, this is the first case of chylothorax occurred due to primary renal lymphoma in literature.

\section{Case report}

A 79-year-old male asymptomatic patient referred to the hospital for routine check-up. He had comorbidities such as hypertension, diabetes mellitus and coronary artery disease. He was an ex-smoker. His physical examination was unremarkable. The serum creatinine value was elevated $(1.69 \mathrm{mg} / \mathrm{dL})$, the full blood count parameters showed normal values. The chest X-ray revealed left pleural effusion (see Figure 1), so thoracentesis was performed to investigate the etiology of pleuresia. Pleural fluid with a chylous appearance had a triglyceride level of $409 \mathrm{mg} / \mathrm{dL}$, which was greater than $110 \mathrm{mg} / \mathrm{dL}$ and supported the diagnosis of chylothorax. The pleural fluid was exudative with lymphocyte dominance; cytology was negative for malignancy.

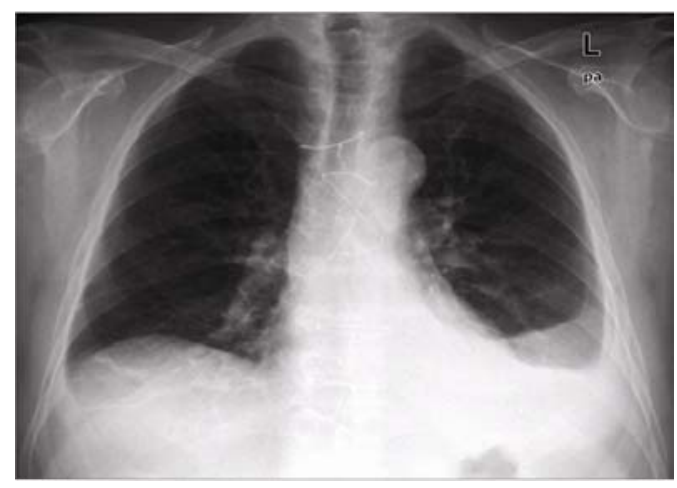

Figure 1. Chest X-ray with left pleural effusion

There was a left renal mass and left pleural effusion without a parenchymal or mediastinal abnormality in thoracoabdominal computurized tomography. Magnetic resonance imaging of the abdomen revealed a mass lesion taking origin from the superior pole of the left kidney with multiple periaortic and perirenal lymphadenopathies, which was interpreted as lymphoma (see Figure 2). The microscopic examination obtained by tru-cut biopsy from renal lesion revealed a diffuse neoplastic lymphocytic infiltration invasing muscle tissue. The immunohistochemical analysis demonstrated a CD 20 and Ki-67 expression positivity. The diagnosis was specified as diffuse large B-cell non-Hodgkin lymphoma (NHL).

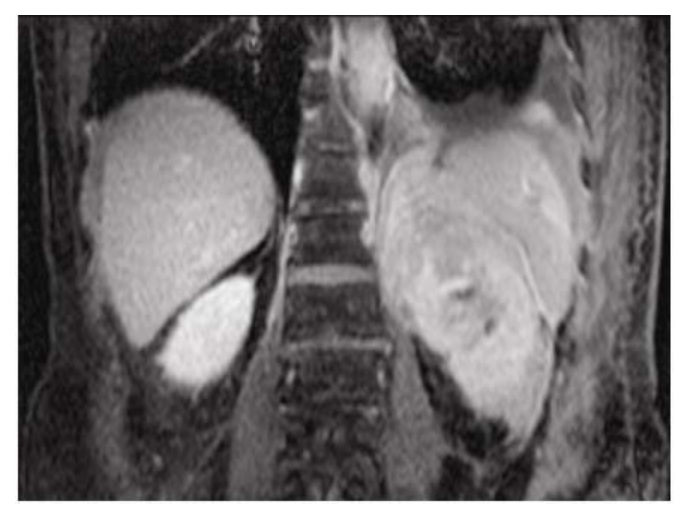

Figure 2. Magnetic resonance imaging of the abdomen with a mass lesion interpreted as lymphoma 


\section{Discussion}

Chylothorax is a rarely seen clinical entity with lymphatic fluid usually characterized by a turbid or white, milky appearance due to a high lipid content, consists of triglycerides, as it is diagnosed when the triglyceride level of pleural fluid is greater than $110 \mathrm{mg} / \mathrm{dL}^{[6]}$.

The etiologies of chylothorax can be categorized as nontraumatic or traumatic. Malignancy is known to be a leading cause of nontraumatic chylothorax, and lymphoma as the most common reason of chylothorax in malignancies with $70 \%-75 \%$ reported in literature ${ }^{[7]}$. On the other hand, Larsen et al. mentioned that; in over 500 cases of lymphoma at their hospital since 1978, there had been only three cases of chylothorax ${ }^{[8]}$. The earlier diagnosis of lymphoma is predicted as the reason of this lower incidence of chylothorax related to lymphoma in series, as chylothorax frequently occurs in patients with advanced disease.

Non-Hodgkin lymphoma (NHL) is a systemic disease generally represents the involvement of any organs or systems. The most common site of genitourinary system involvement is the kidney; primary renal lymphomas (PRL) are rarely seen. Initial extra-nodal presentation of NHL occurs in 10\%-35\% of all patients, and primary renal lymphoma has been reported in only $0.7 \%$ of these cases ${ }^{[9]}$. It is defined as a NHL arising primary in the renal parenchyma, not resulting from invasion of a lymphomatous mass ${ }^{[10]}$.

There are a few theories about the existence of primary renal lymphoma. According to one theory, lymphocytes migrate to the kidney during chronic inflammation, which may start monoclonal proliferation ${ }^{[11]}$. Some authors claim that PRL originates from the renal capsule ${ }^{[12]}$. Also, there are other theories about chronic inflammatory renal conditions and autoimmune mechanisms, which may be possible factors for PRL ${ }^{[13]}$.

Primary renal lymphoma generally represents with a clinical entity such as macroscopic hematuria. A solid mass or involvement in kidney is usually first discovered radiological finding in a patient. This is the first report in literature showing that chylothorax occurs due to primary renal lymphoma. Primary lymphatic disease is not a common cause of chylothorax; however, big mediastinallymphatics or chylous leak can lead to this type of chylothorax ${ }^{[1]}$. As PRL is an uncommon and rare type of malignancy, it is so unusual and atypical for a patient to present with chylothorax.

Primary renal lymphoma, although a rare situation, is an aggressive kind of malignancy which can spread throughout the body at an early period of the disease. This report points out that PRL should be considered in solid renal parenchymal lesions.

As a result, primary renal lymphoma is known to be a rarely seen and an agressive type of NHL. To our best knowledge, this is the first case of primary renal lymphoma which is presented with chylothorax. We would like to report our case with these specialities.

\section{References}

[1] Nair SK, Petko M, Hayward MP. Aetiology and management of chylothorax in adults. European Journal of Cardiothoracic Surgery. 2007; 32: 362-9. http://dx.doi.org/10.1016/j.ejcts.2007.04.024

[2] Valentine VG, Raffin TA. The management of chylothorax. Chest. 1992; 102: 586-591. http://dx.doi.org/10.1378/chest.102.2.586

[3] Doerr CH, Miller DL, Ryu JH. Chylothorax. Semin Respir Crit Care Med. 2001; 22: 617-626. http://dx.doi.org/10.1055/s-2001-18797

[4] Okuno SH, Hoyer JD, Ristow K, Witzig TE: Primary renal non-Hodgkin’s lymphoma. Does it exist? Cancer. 1995; 75: $2258-2261$. http://dx.doi.org/10.1002/1097-0142(19950501)75:9<2258::AID-CNCR2820750911>3.0.CO;2-S

[5] Stallone G, Infante B, Manno C. Primary renal lymphoma does exist: Case report and review of the literature. J Nephrol. 2000; 13: 367-372. 
[6] Perez J, Casal J, Rodriguez W. Always rememberchylothorax. South Med J. 1999; 92: 833-5. http://dx.doi.org/10.1097/00007611-199908000-00020

[7] McWilliams A, Gabbay E. Chylothorax occurring 23 years post-irradiation: literature review and management strategies. Respirology. 2000; 5: 301-3. http://dx.doi.org/10.1046/j.1440-1843.2000.00263.x

[8] Larsen S, Manoharan A, Fermanis G, Schonell M An unusual case of chylothorax complicating non-Hodgkin's lymphoma. Leuk Lymphoma. 2000 Jun; 38(1-2): 207-9.

[9] Anderson T, Chabner BA, Young RC. Malignant lymphoma. The histology and staging of 473 patients at the National Cancer Institute. Cancer. 1982; 50: 2699. http://dx.doi.org/10.1002/1097-0142(19821215)50:12<2699::AID-CNCR2820501202>3.0.CO;2-A

[10] Okuno SH, Hoyer JD, Ristow K, et al. Primary renal non-Hodgkin's lymphoma. An unusual extranodal site. Cancer. 1995; 75: 2258-61. http://dx.doi.org/10.1002/1097-0142(19950501)75:9<2258::AID-CNCR2820750911>3.0.CO;2-S

[11] Saito S: Primary renal lymphoma. Case report and review of the literature. UrolInt. 1996; 56: 192-195. http://dx.doi.org/10.1159/000282839

[12] Stallone G, Infante B, Manno C, Campobasso N, Pannarale G, Schena FP: Primary renal lymphoma does exist: Case report and review of the literature. J Nephrol. 2000; 13: 367-372.

[13] GellrichJ, Hakenberg OW, Naumann R. Primary Renal Non-Hodgkin’s Lymphoma - a Difficult Differential Diagnosis. Onkologie 2002; 25: 273-27.7http://dx.doi.org/10.1159/000064322 